\title{
Gradhiva
}

GRADHIV

Revue d'anthropologie et d'histoire des arts

3 | 2006

Du Far West au Louvre : le musée indien de George

Catlin

\section{Genèse d'une arche américaine pour les Indiens}

Genesis of an American ark for the Indians

\section{Claude Macherel}

\section{(2) OpenEdition}

Journals

Édition électronique

URL : http://journals.openedition.org/gradhiva/90

DOI : $10.4000 /$ gradhiva.90

ISSN : 1760-849X

Éditeur

Musée du quai Branly Jacques Chirac

Édition imprimée

Date de publication : 15 juin 2006

Pagination : 17-37

ISBN : 2-915133-26-3

ISSN : 0764-8928

Référence électronique

Claude Macherel, «Genèse d'une arche américaine pour les Indiens », Gradhiva [En ligne], 3 | 2006, mis en ligne le 10 juin 2009, consulté le 30 avril 2019. URL : http://journals.openedition.org/gradhiva/90 ; DOI : 10.4000/gradhiva.90

Ce document a été généré automatiquement le 30 avril 2019.

(c) musée du quai Branly 


\section{Genèse d'une arche américaine pour les Indiens}

Genesis of an American ark for the Indians

Claude Macherel

Dieu dit à Noé : « La fin de toute chair est arrivée, je l'ai décidé, car la terre est pleine de violence à cause

des hommes et je vais les faire disparaître de la terre.

Fais-toi une arche... » Beréchit/Genèse, 6, 13-14. 
George Catlin, Mu-ho-she-kaw, Nuage-Blanc, 1845. Portrait réalisé sur commande du roi LouisPhilippe suite à la représentation donnée par les lowas au Louvre en avril 1845.

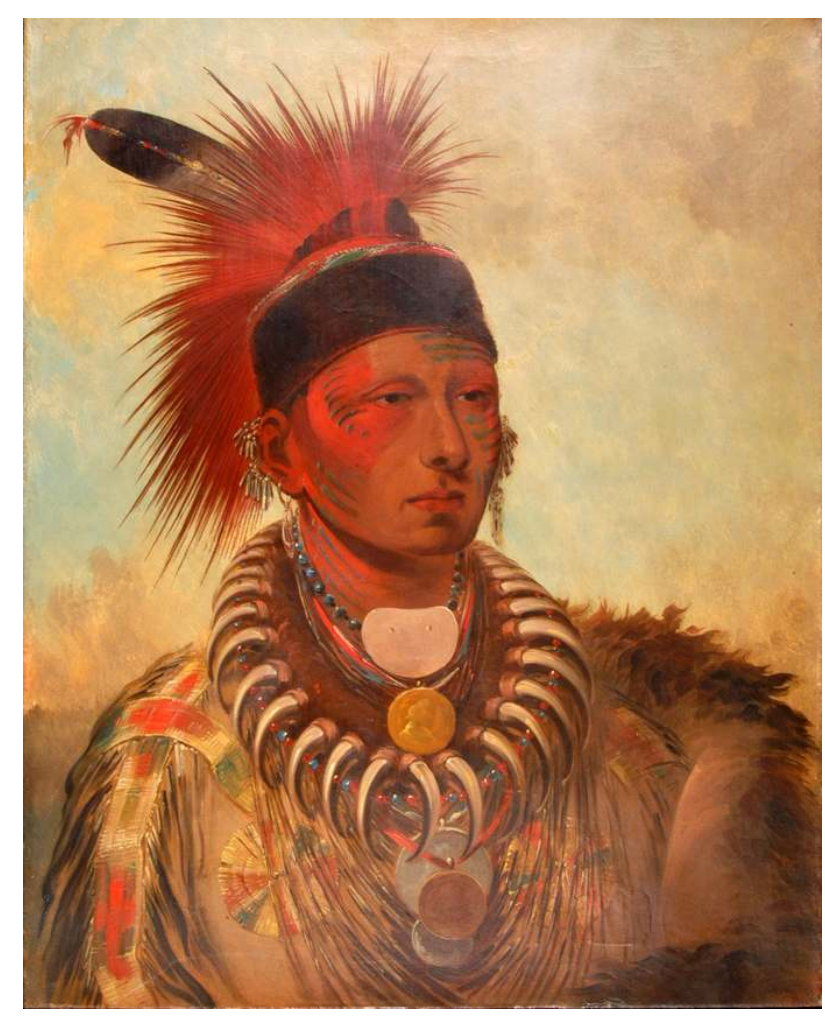

(c) musée du quai Branly

1 Vingt fois posée depuis deux ans au fil de ce travail, la question «Connaissez-vous Catlin ?» reçut deux sortes de réponses : « Euh... non. Qui est-ce ?»; et de temps à autre : «Catlin? ... oui, le peintre américain ». Rassurons ceux qui n'en savent rien. La réponse positive peut s'avérer gagnante dans un dîner en ville, ou alors dans ces compétitions télévisées qui mettent les concurrents au défi de répondre en deux mots.

2 S'agissant de personnes, ces mots sont les premiers qui suivent des noms propres dans une notice de dictionnaire. Deux exemples: "CATLIN (George): Peintre, écrivain et explorateur américain (Wilkes-Barre, Pennsylvanie, 1796 - Jersey City, 1872) ${ }^{1}$. » - « CATLIN, George. American artist and author, whose paintings of Native Americans constitute an invaluable record of their culture in the $19^{\text {th }}$ century ${ }^{2} . »$

Sur le sujet Catlin, je veux dire l'homme, la réponse du " peintre américain », si évidente et sans conséquences qu'elle paraisse, mérite malgré tout examen. Américain, George l'était certainement. Mais en quel sens?

Chacun sait qu'en son temps - la première moitié du XIX ${ }^{\mathrm{e}}$ siècle pour l'essentiel - deux sortes d'Américains vivaient sur le continent : les colons d'un côté, et ceux que Colomb le premier avait cru être « indiens ». Étaient donc "américains » les Anciens d'une part, premiers venus d'Asie, qui avaient intégré à leur humanité, quelques dizaines de millénaires durant, des milieux où faire leur vie, depuis les glaces boréales jusqu'aux terres de feu australes - espaces qu'ils civilisèrent sans en avoir la notion continentale ; les Nouveaux d'autre part, venus d'Europe depuis deux ou trois siècles - ou alors depuis vingt ans seulement, mais ces derniers en très grand nombre. 
5 Puritains anglo-saxons, les colons yankees se tenaient pour des élus de la divine Providence. Ils étaient convaincus d'avoir atteint une Terre promise de leur invention de l'autre côté de l'Atlantique, à l'image des Hébreux Canaan de l'autre côté de la mer Rouge. La traversée d'un océan, l'isolement, la grandeur et la nouveauté du continent, la rupture avec la Couronne britannique enfin, tout transportait les créateurs des États-Unis d'Amérique dans la temporalité mythique d'un recommencement absolu³. Seul obstacle réel à cette négation de l'histoire rationnelle: ils avaient trouvé le Nouveau Monde humainement habité. Comme toujours en pareil cas, les « élus » et leurs élites justifièrent la purification de leur paradis prospectif en niant l'humanité de ses premiers occupants. Assimilés à des bêtes sauvages, "ces animaux qu'on nomme communément Indiens " ${ }^{4}$ furent décrétés "étrangers" à la nation américaine et condamnés de facto à l'extermination, à mesure qu'avancerait la « frontière ».

6 De quel côté de cette frontière d'importation entre Blancs et Indiens, sans cesse repoussée vers l'ouest, situer George Catlin ? L'enquête livre une réponse croisée, cruciale à la lettre. Catlin fut, sa vie durant, des deux côtés de l'Amérique de son temps à la fois. Par naissance, éducation et nécessité, il avait un pied établi à l'Est, où une part de lui-même vécut dans la familiarité et le commerce des Blancs. Ses ancêtres paternels avaient pris pied en Nouvelle-Angleterre dès 1630, dans le sillage du Mayflower. Sa mère et sa parenté maternelle pratiquaient le pacifisme, la solidarité et la tolérance religieuse propres aux Quakers. Lesquels, modérément expansionnistes, entretenaient avec les natifs du voisinage des rapports non violents et le plus possible amicaux. Enfin, son éducation avait pétri Catlin d'une culture européenne, humaniste et classique. Quand les gens de la côte est américaine se dérobèrent, non sans mépris, à ses attentes culturelles, il mit le cap sur l'Europe « aux anciens parapets » avec toute sa création.

7 Son autre pied résidait, par choix réfléchi, dans la familiarité des Indiens. Les ayant rejoints à grand-peine dans l'Occident lointain, le Far West des siens, il vécut des saisons entières près d'eux, aimant leur mode de vie, le partageant tant qu'il put, mu par le désir de connaitre le plus grand nombre possible d'individus et de tribus; acharné ensuite à faire reconnaître leur part d'humanité par la «civilisation» même qui, depuis trois siècles, ne cessait de leur passer sur le corps. Quoi qu'il fitt, en quelque lieu qu'il vécût par la suite, George Catlin emporta partout, dans ses malles ou en lui, ce qu'il avait réussi à capter d'un monde humain voué à disparaître peu après qu'il l'eut pénétré et dépeint.

8 L'hybridation culturelle de Catlin lui est venue de haut et de loin. Le petit George en reçut les empreintes très tôt, de sa mère Polly Sutton, de son père Putnam, et de tout ce qui baigna son enfance entre colons et Indiens. Des colons, faut-il le rappeler, partagés en deux camps qui s'étaient fait la guerre à partir de 1775 ; des deux côtés, des Indiens avaient été enrôlés en guise de légions étrangères, d'autres étaient pris entre les feux des Blancs, jusqu'à la victoire des insurgés yankees et la Déclaration d'indépendance des États-Unis d'Amérique, le 4 juillet 1796.

9 Catlin naquit trois semaines après cette Déclaration, fils et petit-fils de « pères fondateurs de la nation » et de femmes qui avaient couru avec eux tous les risques de la guerre. Ses parents le prénommèrent « George » à l'image de Washington, Father of the Country et son premier président.

10 Des coupures et des sutures à grande profondeur façonnèrent ainsi le petit d'homme. Dans les derniers mots que le vieillard prononça, c'est d'elles et du sort futur de ce que sa vie avait fait d'elles, que Catlin se préoccupait. Par identification réciproque, il avait 
confondu sa vie et son œuvre; il avait soudé le contenu amérindien de celle-ci à son contenant blanc : un musée. Agonisant, il demanda à sa fille Clara : "What will become of my Gallery ? » - «Que va-t-il advenir de mon Musée ? » Son Musée indien, cela va sans dire 5 .

La vie de George Catlin est remarquable en raison de la force peu commune des bouclages qui l'animèrent. La continuité et la cohérence de sa trajectoire impressionnent, tant elle fut secouée, jusqu'au naufrage, de péripéties intenses inlassablement affrontées et surmontées. Les derniers mots de ce navigateur impénitent condensent à l'extrême l'erre et le cap de son parcours.

La vulgate enseigne que Catlin fut avant tout un "peintre", un "artiste» qui, accessoirement, écrivit et voyagea au xIX ${ }^{e}$ siècle en terres indiennes. Ses tableaux, globalement dépréciés de son vivant avant d'être littéralement sauvés des eaux - mais aussi d'une dispersion qui eût détruit l'essentiel, l'unité de son Musée indien - sont déclarés « sans prix » sept ou huit générations après qu'il les eut peints. Que valent cette classification professionnelle et ces jugements?

George Catlin, Les Ojibwa présentent leur danse de guerre à la Cour de la Reine Victoria, château de Windsor, Waterloo Gallery

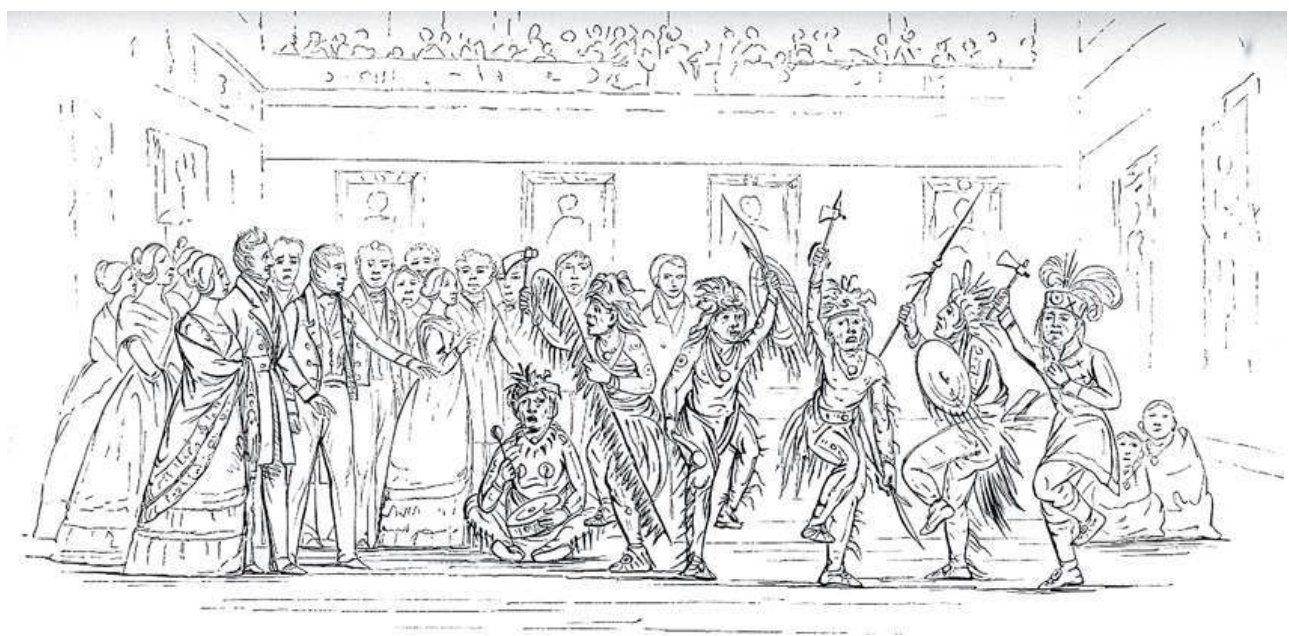

Archives and Rare Books Library, University Libraries, University of Cincinnati

\section{Un peintre... Quel peintre?}

Catlin a certes beaucoup peint, à l'huile où à l'aquarelle ; il a dessiné et gravé sans relâche, des portraits individuels surtout, mais aussi des paysages, des animaux, des scènes à personnages multiples - rituels, danses, chasses, navigations, vie quotidienne, jeux cérémoniels... Il a peint sur ivoire (des miniatures), sur toile ou sur carton. Dans ses travaux gravés et édités, indissociables de ses écrits, il a mis pointe-sèche et burin, eauforte et lithographie au service de la diffusion de ses images. Soit près de 700 miniatures et tableaux en tout, autant de cartoons, et des gravures reproduites à foison.

Ce qui subsiste de tangible de son travail, outre quelques objets muséographiés réchappés de la ruine de son immense collection indienne, c'est en effet sa production picturale et graphique, jointe à son œuvre publiée : livres, portfolios, catalogues, articles de journaux, imprimés de circonstance ${ }^{6}$. 

le Smithsonian American Art Museum, puis présentée dans les grandes villes du pays ${ }^{7}$. Notre ouvrage était sur le métier quand une équipe de chercheurs de l'université de Cincinnati (Ohio) publia une réédition numérisée de quatre textes majeurs de Catlin, imagée de six cents cartoons ${ }^{8}$. Elle comprend le compte rendu narratif des expéditions ethnographiques de Catlin dans l'Ouest américain; un précieux portfolio de scènes de chasse et de jeux indiens; une description sans équivalent de l'o-kee-pa, rite annuel de grande ampleur, pivot de la vie sociale et religieuse des Mandans; enfin, le récit de la migration en Europe de l'Indian Gallery et la narration des péripéties de ses multiples présentations publiques, en Angleterre (1840-1845) puis à Paris, du printemps à l'automne $1845^{9}$.

Jointes aux publications originales des écrits et des gravures en Europe, les expositions de l'Indian Gallery ajoutent une deuxième composante d'identification majeure à la trajectoire de Catlin: si l'œuvre est doublement américaine dans sa genèse et sa réalisation, la connaissance et la reconnaissance de sa valeur furent, de son vivant, très majoritairement européennes.

Cape d'apparat en peau de bison des Indiens des Plaines, sans doute mandan, fin du XIX ${ }^{\mathrm{e}}$ siècle.

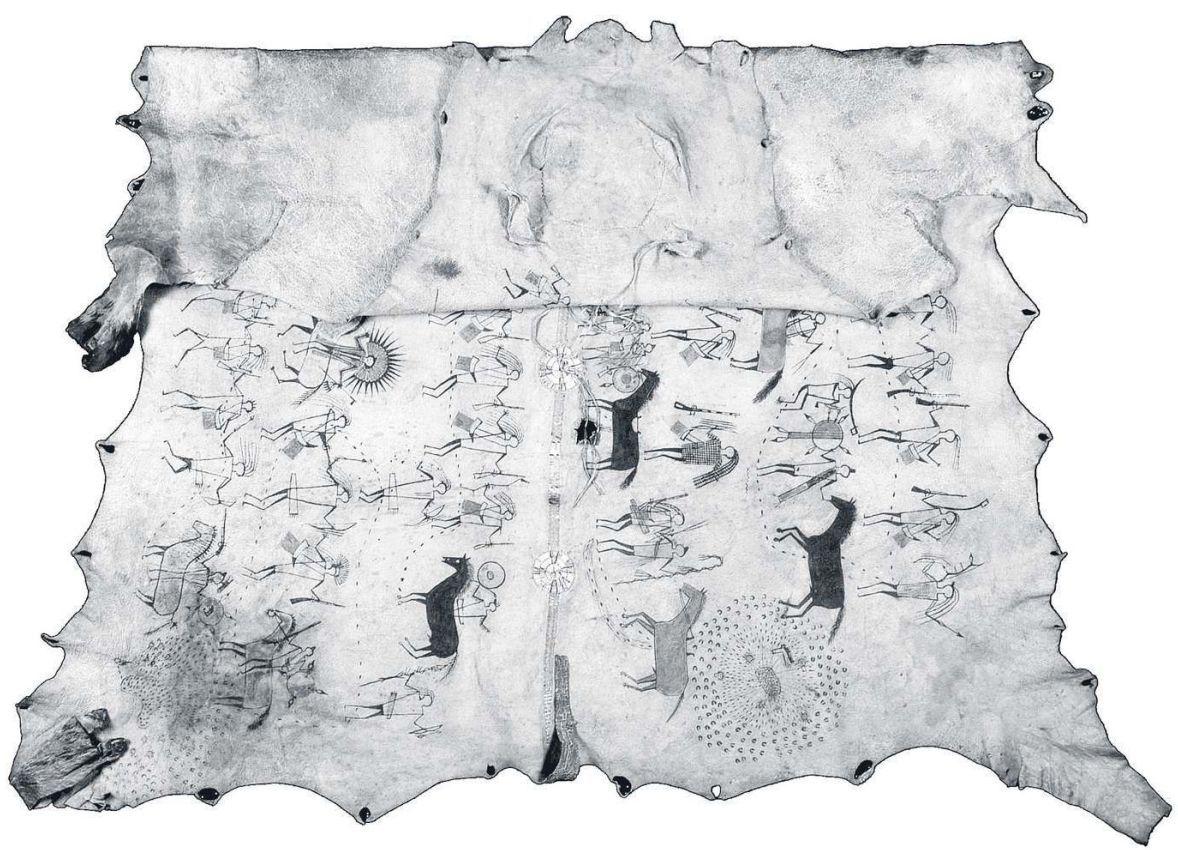

(c) musée du quai Branly 


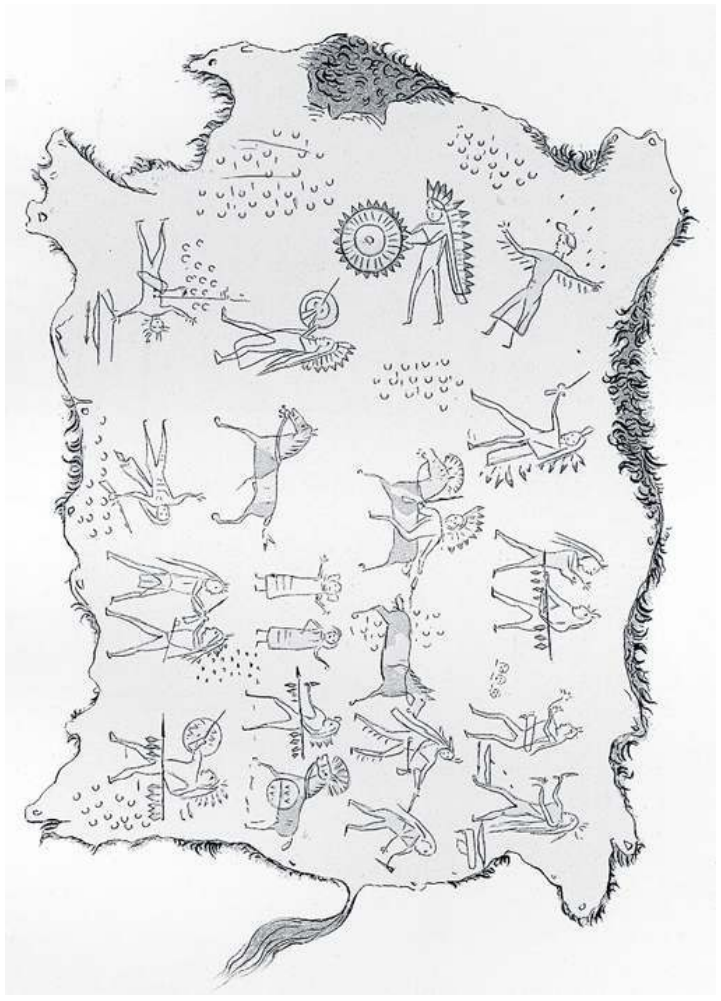

Archives and Rare Books Library, University Libraries, University of Cincinnati

L'activité picturale de Catlin, initiée par le dessin dès l'enfance, reprise et systématiquement poursuivie à partir de ses 25 ans, n'eut jamais pour objectif de lui ouvrir une carrière professionnelle dans le secteur des Beaux-Arts. Nonobstant, les critiques de Catlin et les historiens de son œuvre présupposent comme allant de soi que telle était son ambition ${ }^{10}$. Ils se prévalent alors de ce classement :

1. Pour rappeler que les premières productions catliniennes (des portraits) reçurent de ses pairs en peinture et de la critique un jugement pour le moins mitigé. Ce qui est vrai.

2. Pour emboîter le pas de ces juges un siècle et demi plus tard, et juger eux-mêmes qu'à l'exception de quelques portraits manifestement au-dessus du lot, son métier et son art de peindre étaient très moyens, voire médiocres.

3. Pour conclure enfin, par une déduction qui se donne l'air d'une évidence, que Catlin ayant vu passer, en 1828 à Philadelphie, une délégation de Winnebagos en route pour Washington, trouva là un pis-aller ${ }^{11}$. En substance, voyant sa fortune picturale condamnée par l' establishment régnant, entre Boston et Washington, sans qu'il renonçât pour autant à «se faire un nom d'artiste », il se serait rabattu sur des « sujets » de troisième classe, les Indiens, condamnés eux aussi, mais de plus impitoyable façon, ce dont chacun sur la Côte est était dès cette époque convaincu.

19 L'épreuve des faits dissout ce syllogisme controuvé. Il se trouve qu'en 1817, à l'instigation de son père Putnam, George était allé étudier le droit à Litchfield (Connecticut). Son examen d'avocat passé, il s'était inscrit au barreau. En 1821, las d'occupations bureaucratiques qui le «barbaient », et jugeant sans doute qu'il avait accompli son devoir filial, Catlin vendit tout son attirail juridique pour s'acheter chevalet, godets et pinceaux. Putnam en fut déçu. En bon père qu'il était, il souhaita malgré tout grand succès à son fils, 
lui conseillant d'imiter « Michel-Ange, Rubens, Raphaël, Van Dyck, Rembrandt, Titien, et autres artistes dans ta ligne ${ }^{12}$ ».

C'est sur cette louable et flamboyante ambition paternelle que la critique se fonde pour classer Catlin dans la filiation des grands maîtres; et disqualifier du même coup, avec quelques nuances, sa production picturale. Lui-même n'a jamais prétendu avoir été porté par une ambition de ce genre. Voici son récit de la rencontre de Philadelphie, de son impact et de ses suites:

" Je me demandais continuellement à quelle branche ou à quelle entreprise de l'art je pourrais dédier une vie entière d'enthousiasme, quand une délégation de dix ou quinze dignitaires winnebags venus $\mathrm{du}$ "Far West" sauvage parurent soudain dans la ville, magnifiquement équipés et vêtus : bouclier et heaume, tunique et manteau, teintés et pomponnés, faits à peindre! Silencieux, dignes et stoïques, ces seigneurs de la forêt se pavanèrent dans la ville pendant quelques jours, enveloppés de leurs robes peintes, leur front emplumé des pennes de l'aigle royal [...], après quoi ils s'en allèrent vers Washington. »

21 Catlin songe "longuement et profondément" aux impressions reçues. Avant d'en " déduire et conclure » que «l'habit noir et bleu de la civilisation ne sert pas qu'à voiler, mais aussi à gommer la grâce et la beauté de la Nature. Dans la simplicité et la solitude de leur monde, des hommes qui ne sont pas contraints ni entravés d'artifices, sont certainement les plus beaux modèles qu'un peintre puisse avoir. [...]»

Peindre, cependant, n'est pas le tout de ce qu'il projette de réaliser :

«De plus, l'histoire et les mœurs de tels peuples, conservées par images, sont des thèmes qui valent qu'un homme y dédie sa vie et rien, sauf la perte de ma vie, ne m'empêchera de visiter leur pays et me faire leur historien. Il y avait quelque chose d'inexprimablement délicieux dans cette résolution. Elle me conduirait au milieu de modèles pour mes pinceaux; elle me remettrait en mains du même mouvement, pour mes ressources et ma protection, [le fusil et la canne à pêche] que j'ai chéris enfant ${ }^{13}$."

Ainsi, la rencontre de Philadelphie ne figura-t-elle pas pour Catlin les prémices d'un lot de consolation. L'œil pictural y montre son appétit de matières et de couleurs, celui dont Baudelaire aima tant la restitution, au vu des portraits de Petit Loup et de Graisse de dos de bison, exposés au Salon de 1846 :

"Quand M. Catlin vint à Paris, avec ses Ioways et son musée, le bruit se répandit que c'était un brave homme qui ne savait ni peindre ni dessiner, et que s'il avait fait quelques ébauches passables, c'était grâce à son courage et à sa patience. »

Les rumeurs répandues dans les salons parisiens de 1845 sont l'écho exact à la réputation d'homme courageux, mais peintre laborieux et médiocre, faite à Catlin sept ans plus tôt par les salons de New York et de Philadelphie. Ces bruits pourraient bien avoir traversé l'Atlantique en même temps que Catlin et son musée eux-mêmes, collés à cet homme et à son œuvre par la glu tenace des renommées.

«Il est aujourd'hui avéré, poursuit Baudelaire, que M. Catlin sait fort bien peindre et fort bien dessiner. [...] Quant à la couleur, elle a quelque chose de mystérieux qui me plaît plus que je ne saurais dire. Le rouge, la couleur du sang, la couleur de la vie, abondait tellement dans ce sombre musée, que c'était une ivresse ; quant aux paysages - montagnes boisées, savanes immenses, rivières désertes -, ils étaient monotonement, éternellement verts; le rouge, cette couleur si obscure, si épaisse, plus difficile à pénétrer que les yeux d'un serpent - le vert, cette couleur calme et gaie et souriante de la nature, je les retrouve chantant leur antithèse mélodique jusque sur le visage de ces deux héros ${ }^{14}$.» 
À Philadelphie et conjointement à l'œil fasciné du peintre, un aiguillon descriptif, ethnographique et historique piqua Catlin. La présence et l'allure de ces seigneurs rutilants et fiers agirent sur lui comme un électrochoc. Elles le mobilisèrent tout entier ; elles accrochèrent et revivifièrent son goût très ancien de la vie sauvage; elles ranimèrent des expériences précoces, que le «fusil et la canne à pêche » cernent ici en deux mots. La vue des Indiens paradant paisiblement dans la ville agit comme la révélation de strates enfouies. Le sillage nostalgique de leur passage retraçait les voies anciennes d'une existence qui, loin d'un présent lui convenait comme une toge à un cerf, nouerait le passé chéri qu'il portait en lui au plus désirable des devenirs.

George Catlin, l'artiste chasseur faisant une démonstration de sa carabine Colt à répétition dans un village indien des Caraïbes, vers 1850. Catlin a trouvé en Sam Colt, le fameux fabricant d'armes, un commanditaire de ses voyages sud-américains. Tiré de American héritage, juin 1968.

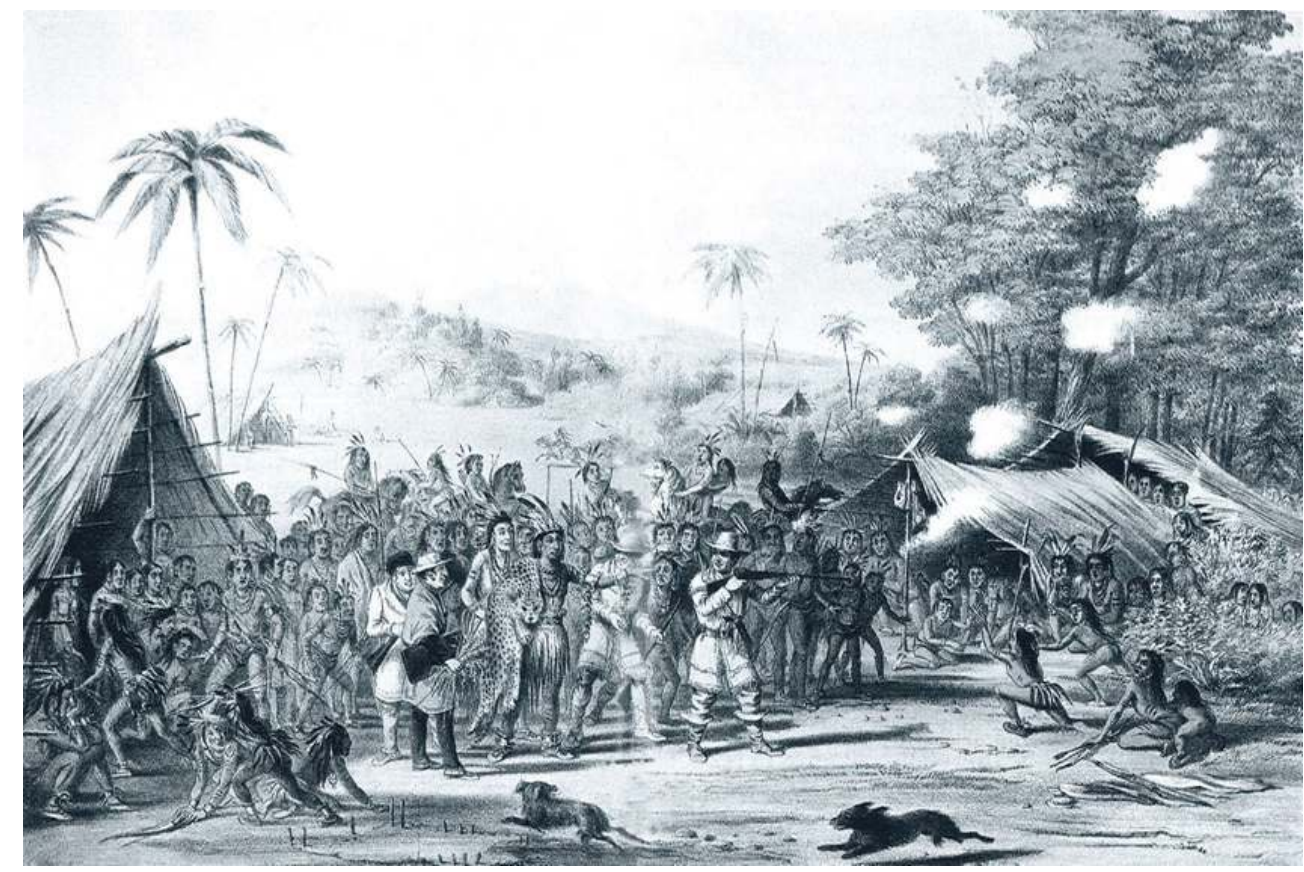

droits réservés

Il prit dès lors la résolution irrévocable de poursuivre une entreprise de connaissance directe de cette portion d'humanité à la fois semblable et différente. L'alliage, rare aujourd'hui encore, est exactement celui dont sont faites les vies de ceux que l'on commençait alors à nommer "ethnographes». Un bon demi-siècle avant que des institutions scientifiques et universitaires ne légitiment la profession, Catlin en discerna la composition en lui avec une lucidité et une prescience remarquables. Puis il y voua sa vie.

\section{Naissance d'une nation}

Cet alliage était ancré dans les expériences matricielles évoquées plus haut. Chose étonnante, personne n'y a prêté attention jusqu'ici. «Il ne reste rien de l'histoire [de Catlin] enfant, si ce n'est quelques notes éparses dans ses propres publications ", écrit Thomas Donaldson, son premier biographe ${ }^{15}$. Quant au dernier en date, Brian Dippie, il ne 
décèle rien qui puisse fonder le tournant décisif de 1828: "On ne sait trop pourquoi Catlin en est venu à portraiturer des Indiens ${ }^{16}$. »

L'érudition catlinienne au complet, édition de Cincinnati comprise, fait curieusement l'impasse sur le plus personnel et le plus vivant des ouvrages de Catlin ; celui aussi qui fut le plus largement traduit et diffusé au XIX⿸e siècle : La Vie chez les Indiens. Scènes et aventures de voyage parmi les tribus des deux Amériques. Ouvrage écrit pour la jeunesse, illustré de 25 gravures ${ }^{17}$.

29 Les parents Catlin, Polly Sutton, née en 1770, et Putnam, né en 1764, ont traversé sans dommage du côté des insurgés, de 1773 à 1783, la guerre d'Indépendance américaine. Sans dommage mais non sans périls. En 1797, Putnam avait abandonné sa fonction d'avocat à Wilkes-Bare et acquis dans l'Ona-qua-gua, superbe région montagneuse sur le cours supérieur de la Susquehanna, un domaine pour partie déjà cultivé, pour partie vierge encore. Il dirigeait l'exploitation, ses domestiques agricoles et sa croissante famille en gentleman-farmer cultivé et compétent, biblique et bienveillant. Les enfants furent nourris des récits épiques entrecroisés que filaient les mémoires parentales : geste de la gestation sanglante d'une nation, que leurs parents et grands-parents avaient activement contribué à mettre au monde.

30 Eli Catlin, le grand-père paternel, avait été parmi les premiers volontaires qui se présentèrent à l'appel de George Washington. Nommé lieutenant en janvier 1776, au $19^{\mathrm{e}}$ régiment d'une armée qui apprenait au feu à faire la guerre, Eli enrôle l'année suivante dans sa compagnie son fils unique Putnam, 13 ans, section des fifres sifflants et des tambours battants : de puissants stimulants collectifs, quand le moment arrive de monter en ligne sans ciller au-devant du feu ennemi ${ }^{18}$. Promu fifre-major, Putnam restera sous les drapeaux jusqu'à la victoire des insurgés. Son congé, rehaussé d'un « badge du mérite » et signé par Washington lui-même, était là, présent dans la maison ${ }^{19}$.

31 La guerre battait son plein et Polly n'avait pas huit ans quand son père, l'un des premiers à s'établir dans la vallée du Wyoming (Pennsylvanie), prit part en juillet 1778 à un combat fameux connu sous le nom du "Massacre de Wyoming» dans la mémoire blanche américaine. Chassés de leurs terres par les colons, les Indiens avaient attaqué par surprise, dans les gorges de la Susquehanna, quelques centaines d'hommes armés qui venaient à eux pour en finir. Leurs arcs et leurs flèches, leurs tomawaks et leurs couteaux à scalper firent des ravages.

Avant de monter à l'assaut, les Blancs avaient mis femmes et enfants à l'abri d'un fort. Polly Sutton et sa mère étaient du nombre. Le fort se trouvant dégarni de sa garnison, les Indiens s'en emparèrent. Cette prise annonçait, a priori, le pire de ce qui pouvait arriver aux femmes. Les hostilités terminées, les Indiens libérèrent pourtant intactes la plupart d'entre elles, emportant seulement quelques jeunes prisonnières dans leur retraite, dont Frances, une fillette de cinq ans, compagne de jeux de Polly. On retrouva Frances très civilement acculturée, cinquante-neuf ans après dans l'Indiana, chez des Miamis qui l'avaient ainsi deux fois conquise : à la guerre et au foyer.

Le père Sutton était sur le champ de bataille. George Catlin tenait de sa bouche un récit du combat, de sa violence, de ses causes et du peu de combattants qui en réchappèrent. Mais son grand-père était heureusement là pour raconter et lui dire comment lui-même, poursuivi par les Indiens victorieux, avait sauvé sa vie en traversant à la nage une rivière sous une pluie de flèches ${ }^{20}$. Oneidas et Mohawks, mis en déroute à leur tour peu après et 
pourchassés par les vengeurs des victimes du massacre, se battirent pied à pied dans leur retraite par monts et par vaux.

\section{Enfance d'une collection} et sans voix.

C'était précisément le long de la Susquehanna, dans les gorges et défilés de l'Ona-qua-gua que des restes ensevelis dans le sol, pendants indigènes de la discharge washingtonienne de Putman et donnant corps comme elle aux récits maternels et grand-maternels, témoignaient de la défaite et du massacre des Indiens en fuite. De sorte que George Catlin put écrire dans La Vie des Indiens, ouvrage écrit pour la jeunesse:

"Les charrues de mon père retournaient chaque jour dans les champs des crânes ou des colliers de verroterie, ainsi que des pointes de flèches en silex, dépouilles indiennes que les laboureurs de la ferme aussi bien que ceux du voisinage ne manquaient pas de m'apporter, et dont je composais, avec une sorte de passion, un petit musée. Un jour, un des laboureurs m'apporta, comme la plus belle et la plus précieuse de ces acquisitions, la partie supérieure d'un casse-tête indien, dont le manche tombé en poussière, avait pourtant laissée intacte, bien que couverte de rouille, la pipe qu'il avait contenue ${ }^{21}$. »

Des récits d'une épopée patriotique d'un côté, les reliques de guerriers sans sépulture, victimes de patriotes chasseurs d'Indiens de l'autre. À la saison des labours nourriciers, la moisson d'ossements, de parures féminines et d'armes de guerre était quotidienne. Le paradoxe tangible, sensible en actes, se doublait de deux télescopages temporels, l'historique irréversible et l'annuel cyclique. Dans la tête du jeune George, tout ce remueméninges devait entraîner un singulier dilemme. Lui-même et les siens vivaient de cultiver sa terre natale; enfouis dans leur sol natal à eux, les restes d'Indiens non moins cultivés surgissaient de la glèbe retournée par les charrues. Sur ce sol, deux moitiés américaines d'humanité s'étaient entretuées ; à présent, dans ses mains de collectionneur comme dans la nourriture des repas familiaux, champ de bataille et champ de blé ne faisaient plus qu'une seule terre. L'envergure des liaisons en jeu, leur densité, les forces qui les tenaient ensemble sont telles qu'il faut nous y arrêter un instant.

Rien n'est plus incompréhensible pour un enfant que la mort. Et peu de choses terrifient autant les petits des hommes qu'un abandon sans reprise ni recours. En conséquence, rien n'attire plus vivement les enfants que les restes, sécrétions mortes de leur propre corps, ou bien ces choses abandonnées des adultes qu'ils trouvent gisantes au sol, inertes

L'enfant recueille ces trouvailles, les relève, les conserve, les modèle, les collectionne, les agence en séries selon sa raison et son cœur. Pour tout dire, il remet en circulation culturelle et en valeur des restes de vie, chaque fois qu'il fait humainement quelque chose d'une dépouille point trop informe, d'un fèces odorant, de débris re-connaissables, si menus soient-ils. Toutes choses trouvées perdues, produits vidés de leur humanité (forme, couleur ou d'autres qualités sensibles exceptées), pour la raison très profonde que voici : personne n'a donné ceci à personne.

Dans la dynamique vitale de l'existence sociale de Catlin, c'en était assez pour que les précieuses collections de l'enfant croissent à mesure qu'il grandirait lui-même «en sagesse et en âge »; jusqu'à se mesurer, le moment venu, à la vastitude de l'horizon derrière lequel les Oneidas survivants avaient disparu sans retour. Et s'il ne restait plus 
un seul Indien à portée immédiate, ce serait les derniers que Catlin adulte irait rechercher coûte que coûte dans l'Occident lointain ${ }^{22}$.

Décrire en temps, faits et lieux, avec tenants et aboutissants, la genèse du petit Musée indien de Catlin aux rives de la Susquehanna, explique sans doute la passion sans mesure, l'énergie et le savoir-faire que cet homme consacra à créer de toutes pièces et de ses mains un considérable Musée indien. Cependant, dira-t-on, ces milliers d'objets que Catlin alla collecter dans une quarantaine de tribus de l'Ouest, ils ne faisaient pas toute la galerie, loin de là. Comment expliquer le cœur de son Musée, les tableaux ? Les portraits surtout, les plus nombreux, et de nos jours comme jadis pour Baudelaire, Delacroix et quelques autres, les plus fascinants, les plus fameux de ces tableaux?

Et puis comment comprendre cette idée bizarre, qu'il eut très tôt, de mettre du vivant dans un musée? Deux grizzlis des Rocheuses pour commencer; puis des Indiens en personne, costumés, grimés à leur mode, emplumés et armés, accommodés en forme de tableaux vivants, mimant en scène dans un décor de tableaux peints, sous une pléiade d'objets confectionnés dans la Prairie, danses, duels, rituels et moments de repos. Un musée n'est pas un cirque, tout de même...

41 L'ouvrage de Catlin "pour la jeunesse » est dédié à la sienne autant qu'à celle de ses lecteurs. Il y rapporte une tranche de vie autrement parlante que crânes, perles de verre et silex. Le récit éclaire les tableaux vivants et les tableaux tout court de l'Indian Gallery les portraits mieux que les autres. Plus : il révèle où, quand, et par quels liens proprement initiatiques, prit corps un attachement passionné aux cultures amérindiennes, liens que Catlin n'eut dès lors de cesse de renouer. Il prodigua à ces hommes, à leurs femmes, à leurs enfants, à leurs façons de faire et de vivre, un mélange d'admiration, d'empathie, de reconnaissance et de sollicitude pathétique qui confine à une identification pure et simple.

\section{Une rencontre inouïe}

« À neuf ou dix ans déjà, je me servais assez habilement d'un léger fusil de chasse à un seul canon que mon père m'avait destiné. Le carnage que je faisais parmi les canards, les cailles, les faisans et les écureuils, m'attirait une certaine considération de la part des chasseurs du comté. Leurs éloges firent poindre en moi une plus haute ambition : tuer un daim. [...] Dans mes visites à une scierie en ruines, j'avais remarqué des traces fréquentes de daims. [...] Tout près de là se trouvait un lick, une mare d'eau salée que les daims visitent aux chaleurs. [...] Je pris la résolution d'y faire l'épreuve de ma bonne fortune (21-23). »

Le projet s'échafaude. Il passe par la possession d'une carabine de bon calibre, une arme dont George ne s'est jamais servi et qu'il n'est pas assez fort pour tenir en joue. Transgressant l'interdit paternel, le gamin s'empare par ruse du gros calibre convoité, gagne le lieu de l'épreuve dans l'après-midi, «le cœur léger et palpitant», et vient se poster derrière une saillie de rocher. Le lick est à portée, vingt pieds au-dessous de sa cachette, il a posé la carabine sur un parapet de roc. Les heures passent et la nuit vient. L'obscurité lui remet en mémoire le récit terrifiant d'un affût, tenu ici même une nuit entière, par un chasseur du voisinage. Il s'apprête à lâcher prise quand un daim parait sur le sentier du lick, « énorme et précautionneux (23-28). »

L'instant décisif approche à mesure que le daim avance. "Des frissons tumultueux se succédaient dans tout mon être, je ne me rappelle pas d'où ils partaient, mais ils 
m'ébranlaient depuis les pieds jusqu'à la tête, par où ils semblaient s'échapper. [...] Tout, excepté moi-même, était prêt pour l'événement. » Le daim entre dans la mare. Catlin le vise une, deux, trois, dix fois. En vain : l'agitation « enveloppait le canon de ma carabine d'une espèce de brouillard » où s'évanouissait le point de mire. Désaltéré, le daim sort du lick et disparaît. Dépit du chasseur en herbe. Mais le daim revient. Catlin, calmé, croit parvenir à viser juste, il va tirer « lorsque, boum ! Voilà que retentit l'explosion et brille la lumière d'une carabine un peu à ma gauche ! » Le daim bondit hors de l'eau, fait dix pas et s'écroule.

Mortel pour le daim, le coup de feu surprise terrasse simultanément le garçon. En son for intérieur, il avait parié pouvoir faire ses preuves de "grand " chasseur ici même sur un daim. Mieux : un chasseur plus adroit, qui avait su cacher son jeu, venait du premier coup d'abattre le daim à sa place.

Cette aventure d'enfance révèle en George Catlin un trait si précocement, si profondément marqué qu'il orienta sa vie entière. Un désir, manifeste en toutes circonstances, de faire croître et de voir grandir au-delà de lui-même ce qu'il était présentement. À dix ou onze ans, cet appétit l'incite à chasser au-delà de ses forces. À l'âge d'homme, des savoirs, des idéaux, intenses comme une possession, et l'ambition corrélative de les réaliser, le pousseront à tout entreprendre dans les grandes largeurs. Prenant constamment des risques à hauteur de cimes, Catlin atteignit nombre de ses objectifs et sa réussite fut éclatante ; à ses propres yeux certainement, bien qu'il eût été seul à juger ses réalisations recevables. Mais il poursuivait trop tôt, pour en saisir le relief et les feux, un horizon trop vaste pour son temps.

Certificat d'authenticité des portraits d'Indiens de George Catlin

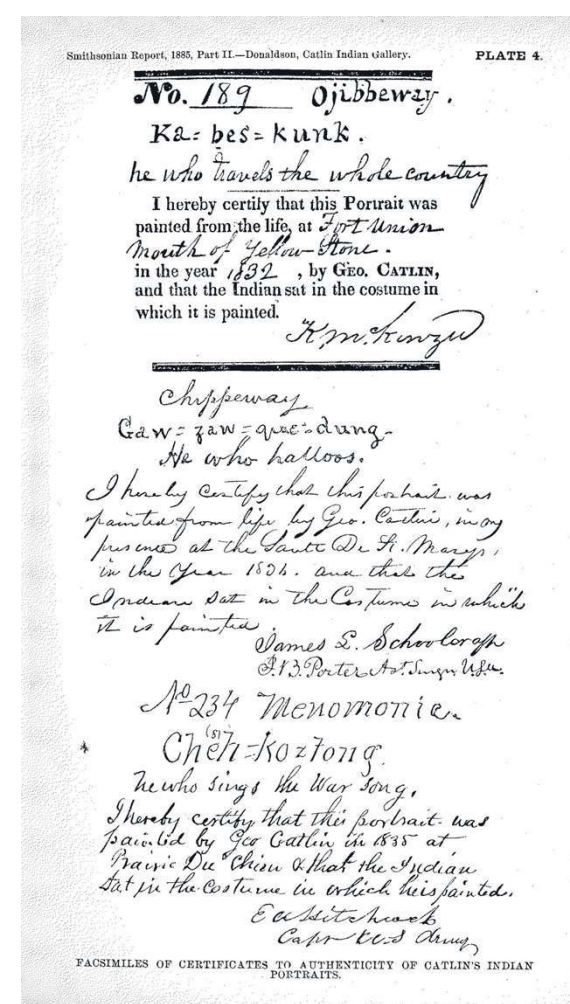

Smithsonian Annual Report II, 1885, smithsonian institution 

George serait-il devenu fou ? Le garçon se réfugie dans son lit en pleurant. Polly, sa mère, vient seule à son chevet. Enfant elle-même, elle a côtoyé de près des Indiens. Elle sait de vive expérience de quoi leur humanité est faite ; égale à celle des Blancs dans la violence des combats, elle en diffère sur un point au moins : au sein de la guerre même, les Indiens se révèlent plus magnanimes aux femmes et aux enfants que les Blancs. "Mon cher George, lui dit-elle, je te crois, je crois que ton histoire est vraie, je crois que tu as vu un Indien (32-35). »

51 Au matin, un laboureur de la ferme annonce qu'il y a « un campement de bohémiens à l'extrémité d'une grande pièce de blé ». «Voilà les Indiens de George!» dit Putnam et, prenant son garçon par la main, il l'emmène au bout du grand champ de blé.

« Nous trouvâmes là mon guerrier Indien, assis sur une peau d'ours étendue sur le sol, les jambes croisées, coudes aux genoux et la pipe à la bouche; à ses côtés se tenaient sa femme et sa petite fille, enveloppées dans des couvertures, le cou orné de colliers de verroterie; devant eux brûlait un petit feu sur lequel cuisait pour le déjeuner une pièce de venaison.

Mon père, qui avait vécu familièrement avec les Indiens, sut chanter leurs chansons, et qui parlait un peu leur langue, me dit : "Tu avais raison mon enfant". 
[...] Je le vis s'avancer vers l'Indien d'un pas ferme et lui tendre la main. J'entendis sortir en même temps de leurs lèvres à tous deux l'amical "How, how, how", et l'Indien me tendit sa main fine et douce pour serrer la mienne (35-36). »

Il comprenait l'anglais et le parlait un peu. « Je suis Oneida, dit-il à mon père, j'habite près du lac Cayuga, à environ cent cinquante miles d'ici ${ }^{24}$, mon nom est On-o-gong-way, grand guerrier.» Ayant invité ses visiteurs à s'asseoir, il bourre à neuf son calumet, l'allume, le donne à fumer à Catlin père, puis le tend au fiston. «Accepte, George, dit Putnam, c'est un témoignage d'amitié. » La rencontre proprement mise en forme, les événements de la veille sont rapportés. Le récit à peine terminé, l'Indien me prit les deux mains en répétant plusieurs fois : «Bon - bon - bon chasseur ! » Puis il se leva, fit quelques pas à l'ombre de la forêt, en rapporta un quartier de venaison qu'il plaça près de moi en s'écriant, sa main appuyée sur ma tête : "Ceci est à vous ! - vous moitié ; - très bon !" "

Du salut en miroir au partage fraternel du gibier, le déroulement de la scène est paisiblement bouleversant pour l'enfant. Mais que fait cet Indien ici? Qu'est-ce qui l'a poussé à faire tant de chemin au péril de sa vie, à travers des contrées habitées ou boisées, à la merci de chasseurs blancs lourdement armés qui tuent les Indiens plus volontiers que des daims?

54 En réponse, On-o-gong-way fit le récit suivant. Son père avait pris part au combat de Wyoming. Les soldats blancs étaient arrivés de là-haut, par les défilés. Un combat eut lieu sur ces champs (il montrait les terres défrichées et mises en culture par Catlin père); ils étaient couverts d'arbres à l'époque. Les soldats firent un grand carnage. Quand une nouvelle troupe de visages pâles survint, les Indiens battirent en retraite.

La parole indienne, on le voit, met les Catlin père et fils, ici, maintenant et autrefois, à une saisissante croisée des chemins. Les combats et massacres dont On-o-gong-way fut témoin, c'est ceux-là mêmes dont la mère et les grands-parents maternels de George réchappèrent sains et saufs. Par un extraordinaire retour de l'un des Indiens survivants et de sa famille sur le champ de bataille, devenu la terre familiale des Catlin vingt et un an plus tard (George avait un an), le garçon entend pour la seconde fois un homme qui y était en dire la violence, mais, cette fois-ci, telle qu'elle fut encaissée de l'autre côté du front.

6 Lui-même, continua On-o-gong-way, n'était alors qu'un enfant, pas plus grand que celuici - il montrait George -, un garçon qui aidait son père à porter des objets enlevés aux Blancs. Parvenus à une crique, ils enterrèrent les objets les plus lourds.

« Je vis mon père et ma mère enfouir une chaudière d'or dans la terre, près du pont là-bas, au pied d'un grand pin qui s'élevait sur la rive. [...] J'ai fait un long chemin pour la retrouver. Mais où irais-je la chercher? Les arbres ont disparu. Nous ne sommes plus qu'un petit nombre, nous vivons très loin d'ici, et nous sommes vos enfants. Mon père ${ }^{25}$, j'ai gardé ce secret pendant des années ; maintenant, le secret n'est plus, il ne cache plus rien et cela rend mon cœur très affligé (40-43). »

Aux questions de Putnam sur la chaudière d'or, On-o-gong-way répondit en formant de ses bras ouverts un grand cercle, qu'il ferma de ses doigts entrecroisés.

- Elle était grande comme ça; elle devait être d'un très grand prix.

- George, dit Putnam, cours à la maison, demande à ta mère de te donner le petit chaudron de cuivre jaune qui est dans la cuisine, et rapporte-le ici.

Quelques années auparavant, un domestique avait déterré un chaudron à l'endroit même indiqué par l'Oneida, sur le bord de la crique. Polly avait intégré la trouvaille à sa batterie de cuisine. 
61 George de retour, et tandis qu'On-o-gong-way considère le chaudron, le retourne en tous sens, Putnam lui explique de quel métal il est fait. L'Indien reste longtemps silencieux, puis «laisse échapper un profond soupir. Après avoir éprouvé la matière avec son couteau, il le déposa, tira sur sa pipe et dit à mon père que c'était bien la chaudière enterrée par ses parents. Deux choses cependant le troublaient fortement : sa petite taille, et que ce ne soit pas de l'or (43-44). »

62 Les jours suivants, George va " passer au petit bivouac presque toutes les heures dont il dispose ». Il apporte sa tête de tomahawk rouillée au guerrier chasseur. On-o-gong-way " lui fait un manche, qu'il perfore de façon très curieuse, en manière de tuyau de pipe ». Comment diable a-t-il fait, se demandent les voisins, vu qu'il n'existe pas de vrille assez longue pour perforer un tube de cette nature? L'Indien donne le secret au garçon: le manche est une tige de frêne ; un tige de métal chauffée ou une baguette d'ébénier suffit pour en extraire la moelle.

63 Catlin va polir le tomahawk reconstruit et affûter son tranchant sur la meule de la ferme. Quand il en revient, « les yeux de l'Indien semblèrent s'allumer, et une lueur soudaine brilla dans les miens quand je le vis bourrer le fourneau de mon tomahawk de cet ersatz de tabac que les Indiens appellent k'nick'neck, et se mettre à fumer cette étrange pipe. [...] Quand la guerre est finie, l'arme meurtrière se change ainsi en instrument de plaisir (45-47)».

64 Annoncée par un coup de feu saisissant, poursuivie par une rencontre médiatisée par Catlin père, consacrée par l'authentique adoubement que fut le partage du gibier unique, généreusement offert par le chasseur accompli à l'apprenti chasseur, l'initiation se poursuit donc, comme le fait toute initiation, par la révélation de savoirs précieux, et donc tenus secrets : ceux qui entrent dans la confection d'une arme redoutable, par exemple, à condition que son possesseur sache aussi s'en servir. Un mystère " encore à apprendre », dit Catlin, et qu'on va maintenant lui dévoiler.

«S'étant levé, il lança le tomahawk en direction d'un arbre éloigné de quelques [mètres]. Il s'y planta. L'Indien recula alors de quinze pas et clik, l'arme vivement lancée s'enfonce une nouvelle fois dans le tronc. Il renouvelle l'exercice plus de vingt fois, sans manquer son but. Les assistants ébahis la voient tourner sur ellemême en fendant l'air et, quelle que soit la distance, le tranchant se ficher profondément dans le bois. »

Ce sont maintenant les silex de sa collection archéologique que George apporte au bivouac. Un fils de ceux qui les ont taillés va remettre à neuf les armes dont ils proviennent. « On-o-gong-way les examina avec attention et une tristesse visible. Il me fit un grand nombre de flèches qu'il garnit de plumes; il courba en un bel arc un jeune ébénier, et l'orna de plumes de grimpereau; avec la peau du faon (sic) que j'avais voulu tuer, il me fit un carquois, y mit des flèches et me le jeta sur le dos (47-48). »

«Mon père leur fit quelques présents, et l'affection que je leur portais et qui m'attirait sans cesse vers eux, préleva sur le garde-manger de ma mère de journalières et larges contributions.

Putnam était dans de constantes appréhensions pour leur sécurité, et, pendant qu'il mûrissait un plan pour les renvoyer à ses frais dans leur pays, par une route différente de celle qu'ils avaient prise en venant, on découvrit un matin que leur fumée ne s'élevait plus au coin de notre grand champ de blé.

Ce matin-là, on trouva appendu, dans une partie ouverte de la maison, un superbe quartier de venaison dans lequel était planté l'une des plus belles plumes d'aigle qui ornait la tête d'On-o-gong-way, et que nous connaissions bien. [...] Preuve de son amitié et de sa gratitude, il avait offert en silence ce présent d'adieu. Ne pouvant 
écrire son nom, il avait chargé la plus belle de ses plumes de faire connaître le donateur.

“Les Indiens sont partis! Les Indiens sont partis!" répéta l'écho pendant cette matinée dans tout le voisinage (48-49).

Quelques jours après le départ des Indiens, deux enfants du voisinage s'exerçaient avec moi au tomahawk. Échappée d'une main peu habile, l'arme effleura l'arbre près duquel je me tenais et son tranchant m'atteignit à la joue gauche, entamant profondément la pommette et me renversant sur le sol, tout couvert de sang. La blessure mit plusieurs mois à guérir, et il m'en reste une cicatrice qui servira de signalement à mes jeunes lecteurs, s'ils ont l'occasion de me voir.

Cette première catastrophe fut suivie d'une seconde, infiniment plus cruelle. Quelques jours plus tard, nous apprîmes que le cadavre d'On-o-gong-way avait été trouvé percé de deux balles de carabine dans la vallée de Randolp, à huit ou dix miles de notre habitation. Personne ne sut ou ne voulut jamais dire quel fut le sort de sa femme et de leur innocente petite fille. Mon père pensait qu'elles avaient été retenues captives, et que l'appât de la chaudière d'or avait été le mobile de cet assassinat (49-50). »

Toute expérience humaine première est initiatique par définition. Il en est d'intimes et comme spontanées. D'autres nouent ces commencements privés à un réseau plus ou moins large, aux mailles plus ou moins serrées, d'actions, d'émotions, d'interactions et de savoirs partagés. Tout ce qu'a vécu George Catlin enfant est frappé de capitales: sa naissance contemporaine de la Déclaration d'indépendance américaine; son identification familiale aux héros paternels et maternels des hauts faits de la guerre d'Indépendance; son identification nominale à leur chef; en amont enfin, des pans entiers de l'histoire de cet autre (re)commencement que fut la colonisation septentrionale du Nouveau Monde, où les siens débarquèrent dès 1630.

Village mandan sur le Haut-Missouri en juin 1832, croquis tiré des carnets de terrain de George Catlin 


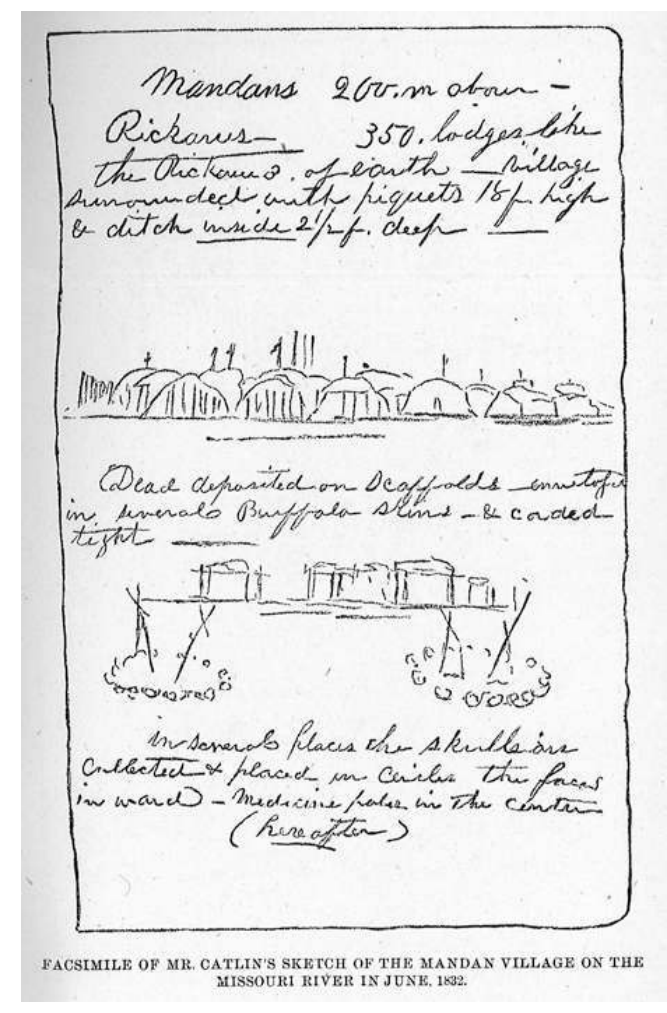

Smithsonian Annual Report II, 1885, smithsonian institution

Des initiales indiennes nombreuses, de grande taille et de fort impact, sont venues très tôt s'entrelacer aux initiales familiales. J'ai déroulé et décrit ici en ethnographe cet entrelacs de deux humanités en un seul homme, comme un biologiste décrit l'écheveau d'un acide aminé, aux deux brins torsadés en sens contraire.

Les deux brins affrontés d'humanité, l'occidental et l'amérindien, que George Catlin porta en lui dès sa petite enfance, son goût de la vie sauvage, ses collections de reliques indiennes et, soudant le tout, le déroulement d'une rencontre inouïe, rendent raison de la genèse en lui de cette création composite, l'Indian Gallery. Il consacra une énergie exceptionnelle à concevoir, charpenter, peupler, mettre et maintenir à flot cette arche voulue pour que survivent, au massacre des Indiens et au laminage de leurs cultures, des portraits, des objets, des images et des descriptions saisies sur le vif, de ce qu'ils avaient vécu, pensé et fait sur leurs terres.

Et si tout le monde donne une place éminente et à part aux portraits peints par Catlin, c'est que ceux-ci doivent beaucoup de leur force d'impact à la matrice d'un visage gravée dans la mémoire de Catlin enfant. Le visage inaugural du chasseur surgi de nulle part et qui, le daim abattu, se retourne vers le garçon, sachant que celui-ci l'observe ; le même visage, à la recherche du chaudron et du temps perdu de son enfance à lui. Enfin, le visage à jamais disparu de On-o-gong-way, parti de son bivouac au coin du grand champ de blé des Catlin en leur laissant, piqué sur un quartier frais de venaison, sa plus belle plume en guise de signature. 


\section{Un prototype de l'Indian Gallery}

70 Du vivant de Catlin, la durée de vie de son œuvre fut très brève. La visibilité et la renommée de l'Indian Gallery au complet durèrent huit ans à peine, de 1838 à 1845 . Il n'empêche : des collections de l'enfant aux derniers mots du mourant, elle occupa sa vie entière. L'identification sans réserve de l'homme à son œuvre est portée par l'enseigne biface qu'il lui a donnée : «L'Indian Gallery de Geo.Catlin ».

71 L'enseigne indique, comme son corollaire en creux, le caractère entier de l'œuvre: la création était toute d'un bloc, insécable. Il a pu copier tel ou tel tableau, jamais il n'a détaillé le musée ; sa création est testimoniale, et un témoignage ne se débite pas en lots. Autre point: l'abréviation de "George» en "Geo.», anodine d'apparence, est remarquable. Ce fut sa signature constante. Qu'entendait-il signifier par là ? Anticipant d'un siècle la photographie aérienne et de 150 ans les satellites photographes, Catlin s'était doté d'une vision globale de la planète: "J'étais soulevé de terre par une paire d'ailes imaginaires. Elles me maintenaient flottant dans l'air, et je pouvais, de là, voir audessous de moi les océans, l'Atlantique et le Pacifique, les grandes cités de l'Est et les fleuves immenses ${ }^{26}$. » En abrégeant le signifiant de son nom baptismal, George a signifié, semble-t-il, le mouvement inverse : l'expansion de ses facultés cognitives à l'échelle d'une planète à décrire: "Geo.» À l'opposé du ciel, la géologie fut d'ailleurs l'une de ses passions. Elle lui a valu de laisser son nom familial à un minéral rare, très recherché des Indiens : une stéatite rougeâtre extraite d'une carrière que Geo. visita, peignit et décrivit en 1836, et dans laquelle un grand nombre de tribus taillaient les fourneaux de leurs calumets. On baptisa le minéral « catlinite » en son honneur.

72 Enfin, Catlin rêva en 1851, peu avant le naufrage de l'Indian Gallery, d'une composition idéale et planétaire, d'une recréation ultime à la mesure de ses ambitions visionnaires : un «musée de l'Humanité », rien de moins, qu'il imagina bien sûr en musée flottant, voguant de port en port ${ }^{27}$. En quoi il ne faisait que transposer sur l'eau une autre caractéristique constante de son musée terrestre : son itinérance.

73 Il n'a pas pour autant tout tiré de son fonds. Outre des conceptions muséographiques communes aux premières décennies $\mathrm{du} \mathrm{xIX}^{\mathrm{e}}$ siècle, que Patricia Falguières inventorie et compare ici même, Catlin a eu sous les yeux le prototype ad hoc d'un musée américain pour les Indiens.

74 Sa résolution prise, en 1828 , de donner une direction neuve et un sens plein à sa vie, Catlin trouva en effet une préfiguration muséale de son projet au département de la Guerre à Washington. Modeste ancêtre du Pentagone, le bâtiment abritait derrière son portique dorique un "Office of Indian Affairs ». Créé en 1817, le bureau fut dirigé par le bienveillant Thomas McKenney jusqu'à son limogeage sec, en août 1830. McKenney recevait là les délégations des nations indiennes convoquées dans la capitale de leur "grand-père » pour y signer des "traités de paix ${ }^{28}$. Ils en repartaient pourvus de vêtements politiquement corrects, voire d'uniformes de l'armée américaine, et d'une bannière de l'Union; porteurs surtout d'une splendide médaille en argent, une «Peace and Friendship Medal ", frappée à l'effigie du président en exercice. Les chefs et les guerriers accordaient le plus grand prix à ce qui était une parure prestigieuse, un gage de bonne foi et de parole donnée tout ensemble - leur "grand-père » en personne ne leur avait-il pas mis la médaille au cou? 
Herman Viola a donné une remarquable vue d'ensemble de l'action indienne du pouvoir américain durant ces années-là ${ }^{29}$. Architecte de cette politique, colombe environnée de faucons, McKenney était aux charnières de l'action, entre une puissance publique très sûre d'elle et des tribus réduites à l'impuissance. Au fil des ans, il avait transformé son bureau en un authentique Musée indien.

Préparant ses missions dans l'Occident lointain, George Catlin n'a pu manquer de fréquenter le département de la Guerre, et spécialement sa pièce indienne. Il conçoit son projet muséal en ce temps-là et commence à le mettre en œuvre peu après que McKenney eut vu son bureau fermé et ses collections dispersées par la toute fraîche administration d'Andrew Jackson. Il engage sa première campagne ethnographique sur le Haut-Missouri au printemps 1830, dans le temps même où Jackson promulgue l'acte définitif de déportation des tribus de l'Est au-delà du Mississippi, le 28 mai 1830.

Le département de la guerre des États-Unis d'Amérique à Washington, dans les années 1820. Sur le plan, en grisé, l'emplacement du bureau de McKenney.

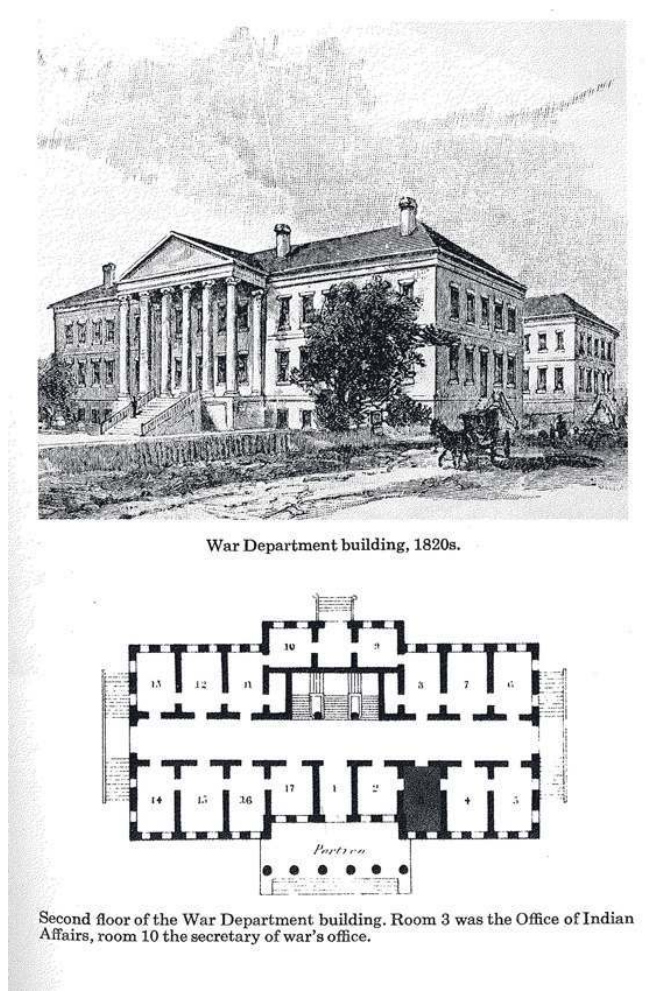

Tiré de Herman J. Viola, Thomas L. McKenney, architect of America's early Indian Policy, 1816-1830, 1974.

Les similitudes entre les réalisations successives de McKenney et de Catlin sont si frappantes que celui-ci a visiblement emprunté au modèle muséal logé au Bureau des Affaires indiennes. Mieux : Catlin refera ce que Jackson a défait, il construira son musée sur les ruines de celui de McKenney. Un an après sa prise de fonction, ce dernier avait commencé à collectionner des objets, à solliciter les agents du bureau en poste sur le terrain, à archiver des documents, à lancer des enquêtes linguistiques, à constituer une bibliothèque. Il ne cessera d'enrichir enquêtes et collections. Vers 1820, il engage en pigiste le portraitiste officieux et appliqué de l'establishment politique, Charles Bird King. Les plus "capés» des délégués indiens viendront poser dans son atelier. Certains 
repartent avec le portrait original, ou avec une copie de taille réduite exécutée à leur intention. En 1843, King aura exécuté en studio 143 portraits, tous au format 17,5 x 14 pouces, tous soigneusement identifiés ${ }^{30}$. Catlin reprendra rigoureusement la formule d'un standard pictural, au format $29 \times 24$ pouces. Lui réalisera 300 portraits sur le terrain. McKenney accrochait les tableaux bord à bord en séries superposées, de façon à tapisser les murs de la pièce. Catlin fera de même.

78 À la fin des années 1820, le 3e bureau du 2e étage du département de la Guerre est tant et si bien indianisé qu'il est devenu attraction touristique. Un guide du district de Columbia, publié par Elliot en $1830^{31}$, recommande vivement aux provinciaux qui visitent la capitale et le Capitole d'aller y faire un tour.

«Les touristes qui suivaient l'incitation d'Elliot [...] vivaient une expérience unique. Un grand canoë d'écorce était suspendu au plafond du vestibule. À l'intérieur du bureau, 130 portraits de dignitaires Indiens décoraient les murs. Au sol, des "curiosités" - vêtements, arcs, pipes, paniers - exposées dans deux vitrines rivalisaient avec le mobilier officiel pour l'occupation de l'espace. Des spécimens minéraux et botaniques, des peaux de bêtes étaient disposés tout autour. Debout à son bureau, McKenney en alerte attendait, prêt à décrire par le menu tout objet présent dans la pièce ${ }^{32}$.»

McKenney avait tenté d'agréger des animaux vivants aux objets inertes : une colombe (sic) en 1826, un élan l'année suivante. L'imitant, Catlin, qui charge sur le Roscius à New York, fin novembre 1839, les sept tonnes de sa galerie pour aller la présenter en Angleterre, embarque deux grizzlis des Rocheuses. Dans leur cage d'acier boulonnée sur le pont du packet ship, les ours apprendront le mal de mer...

80 À Washington, des représentations spectaculaires animent à l'occasion le théâtre des traités. Le gouvernement invite les Indiens à faire parade de leurs talents et convie les citadins au spectacle. Un dimanche de février 1822 par exemple, une démonstration de danses, scènes de chasse et de guerre attire 6000 spectateurs sur la pelouse de la Maison Blanche ${ }^{33}$. Ces spectacles préfigurent ceux que Catlin mettra en scène en Europe, audedans comme au-dehors de l'arche muséale, avec le concours d'Ojibwas et d'Iowas qui furent, sur les planches comme au chapitre financier, ses danseurs : acteurs talentueux de ses succès et agents malgré eux de sa ruine.

81 Aux États-Unis, l'été indien social ménagé par McKenney fut de courte durée. La pression des conquêtes coupa court aux illusions pacifistes. Dès 1827, le secrétaire à la Guerre Barbour demande au surintendant en chef des Affaires indiennes d'arrêter les frais. À ce jour, McKenney a dépensé 3100 dollars d'argent public pour son musée. Un congressiste s'en mêle, «épouvanté » que de pareilles sommes soient dépensées « pour des images de ces misérables, dont il est impossible de dire à quoi elles servent. Je crois qu'elles sont accrochées dans le bureau de $\mathrm{M}^{\mathrm{r}}$ McKenney à Washington pour satisfaire la curiosité des étrangers ${ }^{34} »$.

82 McKenney défend son lieu de mémoire dans deux lettres ouvertes publiées en mai 1828. Son argumentaire est en tous points remarquable d'humanité et d'une intelligence que nous dirions anthropologique :

"Conserver une image ressemblante de peuples pleins d'intérêt est un grand objet, mais ce n'est pas le seul. [...] Voici le principe civil - the policy - de la chose : les Indiens sont semblables aux autres gens à beaucoup d'égards - et ne sont pas moins sensibles que nous le sommes aux marques de respect et d'attention ${ }^{35}$."

83 Catlin date sa conversion à l'indianité de 1828. Son attitude foncière à l'égard des Indiens est exactement accordée à la position humaniste de McKenney. Son lieu de mémoire 
reproduit l'éphémère musée du département de la Guerre, à ceci près que Catlin l'amplifie, meuble son arche indienne de ses mains dans la Prairie même, l'expose dans des salles de location, le tout de bout en bout à ses frais. Au reste, la méconnaissance et le mépris officiels pour les deux œuvres se valent. C'est en vain que Catlin tenta sans relâche, trente-cinq ans durant, de convaincre l'État américain d'acquérir son mémorial indien. "Tout ça ne vaut pas un clou ", rétorqua en substance le Congrès, et pour les Indiens en peinture, l'État a déjà donné.

Au bon temps de la générosité de l'État, dans la décennie 1820, de l'histoire est en train de se faire dans l'espace d'une pièce du ministère de la Guerre et autour d'elle, sur le théâtre de l'exercice du pouvoir. Les chefs des nations indiennes, revêtus des attributs éclatants de leur être social, défilent les uns après les autres, reçus, logés, honorés des semaines ou des mois durant par le gouvernement des Blancs comme s'ils étaient des chefs d'État souverains, aveuglés sur la mascarade qu'on leur fait jouer, puisque celle-ci n'a d'autre objet que de les dépouiller de leurs terres et d'eux-mêmes. Dans les mains, les écrits et les récits de l'artiste ethnographe Catlin, tout ceci deviendra dès 1833, puis en Europe à partir de 1840, une transposition à grande échelle, authentique et visitable, de l'espace même qui était, à Washington, le lieu où, pour la paix des consciences blanches, cette histoire avait été scellée en droit.

Forme et contenu indissociables, ces conservatoires sont des arches décalquées d'un modèle culturel littéralement génétique, celui de Genèse, $6:$ une pièce rectangulaire aux parois couvertes de portraits reconnaissables, pleine d'objets indiens, vibrante du désir de produire un effet de présence, et centrée sur l'homme qui a façonné ce lieu, a réuni les figures et les objets. Lui connaît assez ces hommes et ces choses pour en parler longuement et composer éventuellement, avec le concours d'Indiens de fortune vêtus et équipés de pied en cap, des tableaux vivants de ce qui meurt et va disparaître sans retour du monde réel des vivants ${ }^{36}$. 
Bivouac d'Indiens, gravure de George Catlin, La Vie chez les Indiens, ouvrage écrit pour la jeunesse, 1866.

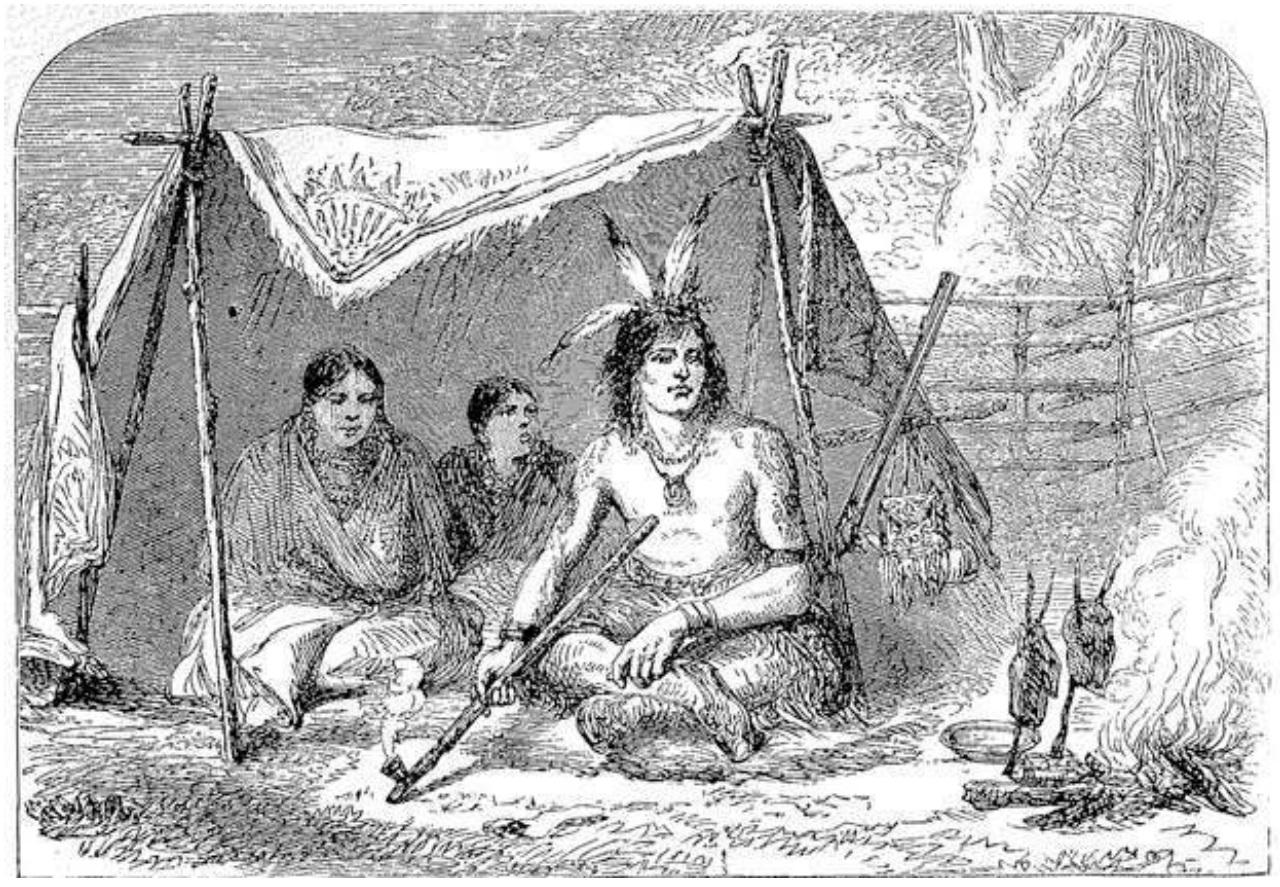

droits réservés

\section{« Now, I am George Catlin again »}

Le samedi 31 octobre 1868, Francis, le benjamin des quatorze frères et sœurs de George Catlin, embarque à midi sur le City of Boston, un packet ship amarré à New York. George, son aîné de vingt ans, l'appelle en Europe. Il est parti dix jours plus tôt de chez lui à Hudson, un bourg pionnier du Wisconsin. En passant par Great Bend (Indiana), il a laissé à son fils Charley sa montre de gousset, en gage d'une avance de 100 dollars. La traversée de l'Atlantique lui coûtera 40 bucks, retour compris. Le solde constitue tout son pécule pour deux mois d'Europe.

Francis débarque à Liverpool le 12 novembre. Il est à Londres le lendemain soir, gagne la côte sud en chemin de fer, passe la Manche dans la nuit, sommeille deux heures sur un banc à Ostende et prend le premier train du petit matin. Une demi-heure plus tard, il descend à Bruxelles gare du Nord. George l'y attend. Les deux frères ne se sont pas revus depuis vingt-neuf ans ${ }^{37}$.

Le 25 décembre 1868, c'est Noël à Bruxelles, Christmas pour les frères Catlin. Dans un deux-pièces hôtelier du faubourg populaire de Saint-Joss, derrière la gare du Nord, Francis regarde George travailler. Il s'est attelé trente mois plus tôt à la réalisation d'une impressionnante série de dessins. Frappé de surdité, pauvre comme Job, il demeure l'homme que la forge entière de sa vie a trempé : Le Peintre des Indiens. Ce dessin-là est le dernier de la série. Il en ponctue la signature à la pointe du crayon, avant de prendre son frère à témoin.

89 Le soir venu, penché sur son journal de voyage, Francis note l'empreinte fraîche de la scène : «Geo a terminé les Dessins [Cartoons] - la dernière marque a été le point à la fin de 
son nom. Il a dit que, de toute sa vie, il n'avait jamais été aussi content de voir un travail quitter ses mains [to get a job off his hands] -, au total, deux ans et demi passés sur ces dessins. Si heureux qu'il semblait être, moins de 5 minutes plus tard il avait mélangé des couleurs, ses pinceaux étaient devant lui. Maintenant, dit-il, je me sens naturel pour la première fois depuis que j'ai commencé les dessins ${ }^{38}$. »

Francis s'interrompt, lève la plume, se ravise. La phrase exacte qu'a prononcée son frère à cet instant était frappante. Elle claque encore dans sa tête. Il la fixe en note : « Now I am Geo. Catlin again, look out for the paint !» - « Maintenant je suis Geo. Catlin à nouveau, tu vas voir la peinture ${ }^{39}$ !»

Entre 1860 et 1870, tandis qu'il refaisait son musée sur cartons dans un deux-pièces de l'hôtel du Duc de Brabant, à Saint-Joss, Catlin nourrissait des souris blanches, passant tous les jours une heure à jouer avec elles ${ }^{40}$. Comme si la présence d'êtres vivants, et une part ludique de liberté, étaient nécessaires à l'arche de l'Indian Gallery, ce modèle réduit d'un monde voué à disparaître qu'il avait patiemment construit de ses mains ${ }^{41}$, animé de spectacles et de spectateurs quelques années durant, puis perdu. Il le recréait ici pour la dernière fois, à plus petite échelle encore, avec pour toute compagnie d'inoffensives miniatures des deux énormes grizzlis qu'il avait embarqués sur le pont du Roscius vingt ou trente ans plus tôt, en même temps que son musée au complet.

\section{BIBLIOGRAPHIE}

BAUDELAIRE, Charles

1976 (1846) CEuvres complètes vol. II. Paris, Gallimard, « Bibliothèque de la Pléiade ».

CATLIN REHM, Marjorie

1966 The Letters of George Catlin and his Family. A Chronicle of the American West. Berkeley, University of California Press - Londres, Cambridge University Press.

CRAWLEY, Ernest

1912 « Drums and Cymbals » Encyclopedia of Relgion and Ethics, vol. 5 : 89-94.

DIPPIE, Brian W.

2002 « Green Fields and Red Men », in Gurney et Heyman, éds., George Catlin and his Indian Gallery. Washington, Smithsonian American Art Museum : 26-61.

DONALDSON, Thomas

1886 The George Catlin Indian Gallery in the U.S. National Museum, with Memoir and Statistics, Annual Report of the Board of Regents of the Smithsonian Institution, juillet, Part II, Washington, Government printing office, VII.

ELLIOT, Jonathan 
1830 Historical Sketches of the Ten Miles Square Forming the District of Columbia ; with a picture of Washington, Describing Objects of General Interest or Curiosity at the Metropolis of the Union.

Washington.

FABRE, Daniel

2003 Le Savoir des différences. Histoire et sciences des mœurs en Europe, $\mathrm{XVIII}{ }^{e}$-XX ${ }^{e}$ siècles, programme de recherche pour l'ACI « Histoire des savoirs », site web du LAHIC, 2005.

FALGUIÈRES, Patricia

2000 « Noé muséographe. Note sur la culture de la curiosité, à Rome, au XVII siècle », in Christoph L. Frommel et Elisabeth Sladek, éd., Francesco Borromini, Atti del convegno internazionale Roma, 13-15 gennaio 2000, Electa : 335-341.

GURNEY George et HEYMAN Therese Thau, éd.

2002 George Catlin and his Indian Gallery. Washington, Smithsonian American Art Museum.

HABERLEY, LOYd

1948 Pursuit of the Horizon. A Life of George Catlin Painter and Recorder of the American Indians. New York, The MacMillan Company.

HASSRICK, Royal B.

1977 The George Catlin Book of American Indians. New York, Watson-Guptill.

LÉVI-STRAUSS, Claude

1962 La Pensée sauvage. Paris, Plon.

MACHEREL, Claude

1984 « L'eau du glacier », Études rurales 93-94 : 205-238.

2002 « La navigation des nefs de pierre chez les Chrétiens occidentaux », Collegium Budapest, Institute for Advanced Studies, site web.

MARIENSTRAS, Élise

1976 Les Mythes fondateurs de la nation américaine. Paris, Maspéro.

MCCRACKEN, Harold

1959 George Catlin and the Old Frontier. A Biography and Picture Gallery of the Dean of Indian Painters. New York, Bonanza Books.

MULVEY, Christopher

2002 « George Catlin in Europe », in Gurney et Heyman, éds., George Catlin and his Indian Gallery. Washington, Smithsonian American Art Museum : 62-91.

NEEDHAM, Rodney

1967 «Percussion and Transition » Man, vol. 2 (4) : 606-614.

PANOFSKI, Erwin

1992 [1951] Architecture gothique et pensée scolastique, traduction et postface de Pierre Bourdieu. Paris, Éditions de Minuit.

SAINT-VICTOR, Hugues de

ca. 1135 De Arca Noe, Patrologia latina vol. 176. 
TROCCOLI, Joan Carpenter

2002 « Illustrated Commentary », in : Gurney et Heyman, éd., George Catlin and his Indian Gallery.

Washington, Smithsonian American Art Museum : 92-247.

UNIVERSITY OF CINCINNATI, éd.

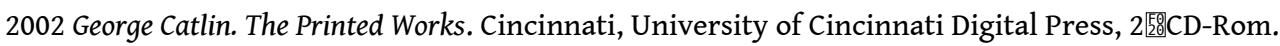

VIOLA, Herman J.

1974 Thomas L. McKenney. Architect of America's Early Indian Policy : 1816-1830. Chicago, The

Swallow Press.

1976 The Indian Legacy of Charles Bird King. Washington, Smithsonian Institution Press, et New York, Doubleday \& Co.

\section{NOTES}

1. Le Petit Robert des noms propres, éd. 1999.

2. «Artiste et auteur américain dont les peintures d'indigènes américains constituent un enregistrement inestimable de leur culture au XIX ${ }^{\mathrm{e}}$ siècle » (www.britannica.com).

3. Marientras 1976 : 61-88.

4. Brackenridge, Indian Atrocities : 71-72 (cité par Marientras 1976 : 166). Franklin, Jefferson et d'autres idéologues du $\mathrm{XVIII}^{\mathrm{e}}$ siècle partageaient des conceptions similaires. Ayant imputé aux Indiens une sauvagerie diabolique et jugé pareillement criminels tous les autres colonisateurs européens, les pionniers anglo-saxons se retrouvaient seuls purs au tribunal de leur puritanisme, uniques porteurs recevables du flambeau régénérateur de «la civilisation (Marientras: 184-208).

5. Thomas Donaldson a recueilli ces mots peu de temps après, de la bouche de Clara Gregory Catlin (Donaldson 1885 : 716-717).

6. Daniel Fabre en a dressé l'inventaire complet sous le titre « Catlin : une bio-bibliogaphie », qui n'a pu trouver place ici. Il est consultable sur www.lahic.cnrs.fr/ateliers, référencé Atelier Catlin, $\mathrm{n}^{\circ} 3$ bis, août 2004 .

7. Dippie, Heyman éd. 2002.

8. Univ. of Cincinnati Digital Press 2004.

9. Catlin $1841,1844,1867$ et 1852 respectivement.

10. Entre autres, Dippie $2002: 30$.

11. Hassrick 1977 : 20-21 ; Dippie 2002 : 29-30. Ardents à faire l'éloge de leur héros, McCracken (1959 : 22-25) et Catlin Rœhm (1966:22-30) gomment ces ombres du tableau.

12. «[...] and other artists in your line». Lettre de Putnam à George, 26 mars 1821, AAA, rouleau 2136 , citée par Dippie $2002: 29$.

13. Manners... : 19-20. (éd. de 1860). Catlin a désigné « [...] le fusil et la canne à pêche » à la page précédente. Il dit ici : « [...] les objets mentionnés plus haut ».

14. Baudelaire (1846) 1976 : 446. Voir l'article de Daniel Fabre.

15. Donaldson 1885 : 704.

16. Dippie $2002: 30$.

17. Catlin 1866. Seul Haberley (1948[医: 3-22) emprunte à ce livre pour la jeunesse, sans s'y référer précisément. Il cite longuement la rencontre qui nous intéresse, sans rien en faire de plus qu'un épisode dramatique et mouvementé, bien accordé à l'écriture romancée de sa "vie de George Catlin », magnifiquement intitulée Poursuite de l'horizon. 
18. Les effets physiques et psychiques du tambour battant sont « complets » (Crawley 1912: 91) et «inévitables », dit justement Needham (1967: 610), dans un texte d'une grande acuité sur l'association, quasi universelle, de percussions aux transitions naturelles socialement mises en forme. Pour une analyse de la portée physique et culturelle de sonneries de cloches, voir Macherel 1984 : 222-227.

19. La famille conservait encore la précieuse décharge un siècle après sa délivrance (Donaldson $1885: 702)$.

20. Catlin, cité par Donaldson $1885: 704$.

21. Catlin 1866: 20-21. "La pipe» (anglais : pipe). Quelques pages plus loin (45-46, nous y viendrons), Catlin décrit avec précision la technique d'emmanchement et les deux emplois d'un tomahawk.

22. Les derniers (des hommes, des Mohicans, des locuteurs d'une langue, etc.) tiennent une place cruciale dans toute réflexion sur le devenir des sociétés humaines. Dans le cadre d'une recherche collective sur la constitution des savoirs ethnologiques en Europe, Daniel Fabre (2003) a développé sur le sujet des analyses pénétrantes.

23. Ici comme dans toutes les citations suivantes, les italiques sont celles de Catlin lui-même.

24. Au sud du lac Ontario, à l'ouest de l'État de New York. On-o-gang-way, sa femme et leur fillette ont marché $240 \mathrm{~km}$ pour revenir dans l'Ona-qua-gua.

25. L'homme s'adresse ici à Putnam, selon l'usage indien de considérer les Blancs comme leurs parents protecteurs, et eux-mêmes comme des enfants qui ont beaucoup à recevoir de leurs ascendants. Ils nommèrent longtemps " grand-père » le président qui siégeait à Washington.

26. Cité par Troccoli 2002: 153. Une gouache magnifique de 1827 présente une vue du ciel, mentalement et picturalement construite au sol, des chutes du Niagara et de leurs alentours (Dippie éd. 2002 : 36). Pour l'Indian Gallery, il avait réalisé un modèle réduit des chutes en trois dimensions (Troccoli, ibid.).

27. Catlin 1851. Voir les extraits de ce texte étonnant publiés ici.

28. Signer seulement: toutes les négociations, sauf cinq, furent traitées sur le terrain (Viola 1974 : 117).

29. Viola 1974 : 116-134 (délégations indiennes à Washington) ; 237-250 (le bureau fait musée) et 222-236 (le limogeage du surintendant).

30. Viola 1976 et $1974: 246-250$.

31. Elliot $1830: 165$.

32. Viola $1974: 238$.

33. Ibid. : 241 (les animaux et les démonstrations spectaculaires).

34. Viola $1974: 248$.

35. Cité par Viola : 1974.

36. Vers 1135 à Paris, dans l'effervescence théologique qui prépare et annonce l'édification des impressionnantes nefs de salut gothiques, Hugues de Saint-Victor compose, avec son De Arca Noe (Patr. Lat. 176), l'élaboration métaphorique la plus grandiose jamais donnée du modèle salvateur de l'arche biblique. Sur les architectures jumelles des sommes et des cathédrales, voir Panofski 1992. Sur la métaphore, salvatrice en rites, des nefs de pierre chrétiennes, voir Macherel 2002. Au $\mathrm{XVII}^{\mathrm{e}}$ siècle, collectionneurs de «curiosités » et architectes muséographes redonneront corps à la métaphore biface de l'arche de Noé, bâtiment et classification liés (Falguières 2000).

37. Catlin Rœhm 1966 : 347-354. Marjorie, suivie par Mulvey (2002:[6078), date la photo de son grand-père « des années 1870 » (332 h.t). Or à Bruxelles, peu avant la Noël de 1868, Francis note : «Ouragan la nuit passée. [...] Beau temps aujourd'hui. Ai posé pour ma photo - pas bonne, je pense. » (ibid., p. 369) C'est vraisemblablement le seul portrait publié connu.

38. Catlin Rœhm $1966: 369$.

39. Ibid.

40. Francis Catlin, « Journal du voyage en Belgique », Rœhm Catlin $1966: 356$ et 367. 
41. «La question se pose de savoir si le modèle réduit, qui est aussi le "chef-d'œuvre" du compagnon, n'offre pas, toujours et partout, le type même de l'œuvre d'art. » (Lévi-Strauss 1962 : $34)$.

\section{RÉSUMÉS}

George Catlin, «peintre américain »? Sans doute, mais en quels sens ? De son temps (1796-1872), deux sortes mutuellement exclusives d'Américains vivaient sur le continent : les Indiens natifs et des colons d'origine européenne. Dès l'enfance, Catlin s'est trouvé au confluent des deux courants. Yankee par filiation, éducation, tempérament, il reçut très tôt l'apport décisif des sources indiennes. Le garçon ne se laissait pas partager. Son histoire et celle des siens, nouées à l'enfantement sanglant de la nation américaine, le mirent donc au défi de concilier des contraires. Catlin releva le défi, l'âge d'homme venu, tenant le pari fou de tout réconcilier dans une création issue de sa tête et de ses mains : «son » Indian Gallery. L'œuvre réalisée, il la fera vivre à se ruiner, identifié à elle jusqu'à son dernier souffle. Le contenant est occidental ; c'est une arche de mémoire. L'Indian Gallery remodélise l'arche mythique de la Genèse, pour sauver de la plus fatale des disparitions, l'oubli, son précieux contenu: de grands pans de cultures indiennes, des milliers d'Indiens en images, autant d'objets, des visages et des usages que la déferlante conquérante de l'Ouest allait engloutir peu après. Mais la peinture ne dépeint pas cet homme tout entier. Indépendant et volontaire, avide de renommée mais piètre commerçant, Catlin était aussi chasseur, navigateur, ethnographe, écrivain, muséographe, metteur en scène de tableaux vivants, des vivants amérindiens, naturellement.

George Catlin, "American painter"? Certainly, but in what sense exactly? During his life (1796-1872), two mutually exclusive types of Americans inhabited the continent: the native Indians and the colonists of European origin. From his earliest childhood, Catlin found himself caught between the two. Yankee by birth, education and temperament, he was decisively influenced very early on by Indian cultures. The boy refused to be divided. His history and that of his family, bound up with the bloody birth of the American nation, set him the challenge of reconciling opposites. The adult Catlin took up the challenge, making the mad gamble of attempting to reconcile everything in a single creation, his own brainchild and the work of his own hands - his Indian Gallery. Once it was completed, he ruined himself to keep it alive, identified with it to his very last breath.

The contents were western ; it was an ark of memories. The Indian Gallery was a recreation of the mythical ark of Genesis, built in order to save its precious contents from that most final of disappearances, forgetfulness: the great vistas of Indian cultures, thousands of Indians captured in paint, as many artefacts, faces and customs that the relentless conquerors of the West were soon to engulf. But the paintings are not the whole man. Independent and headstrong, eager for fame but a poor businessman, Catlin was also hunter, sailor, ethnographer, writer and director of tableaux vivants - with Amerindians in the starring roles, naturally enough. 
INDEX

Keywords : children's collections, identifications, initiation identifications, Noah's Ark, scale models

Mots-clés : arche de Noé, collections enfantines, identifications, initiation, modèles réduits

\section{AUTEUR}

CLAUDE MACHEREL

Chargé de recherche au CNRS, LAHIC, claude.macherel@ivry.cnrs.fr 